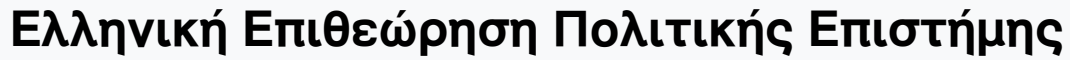

Tó .20 , Ap. 1 (2002)

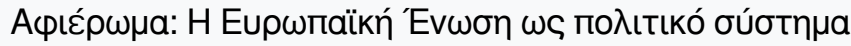

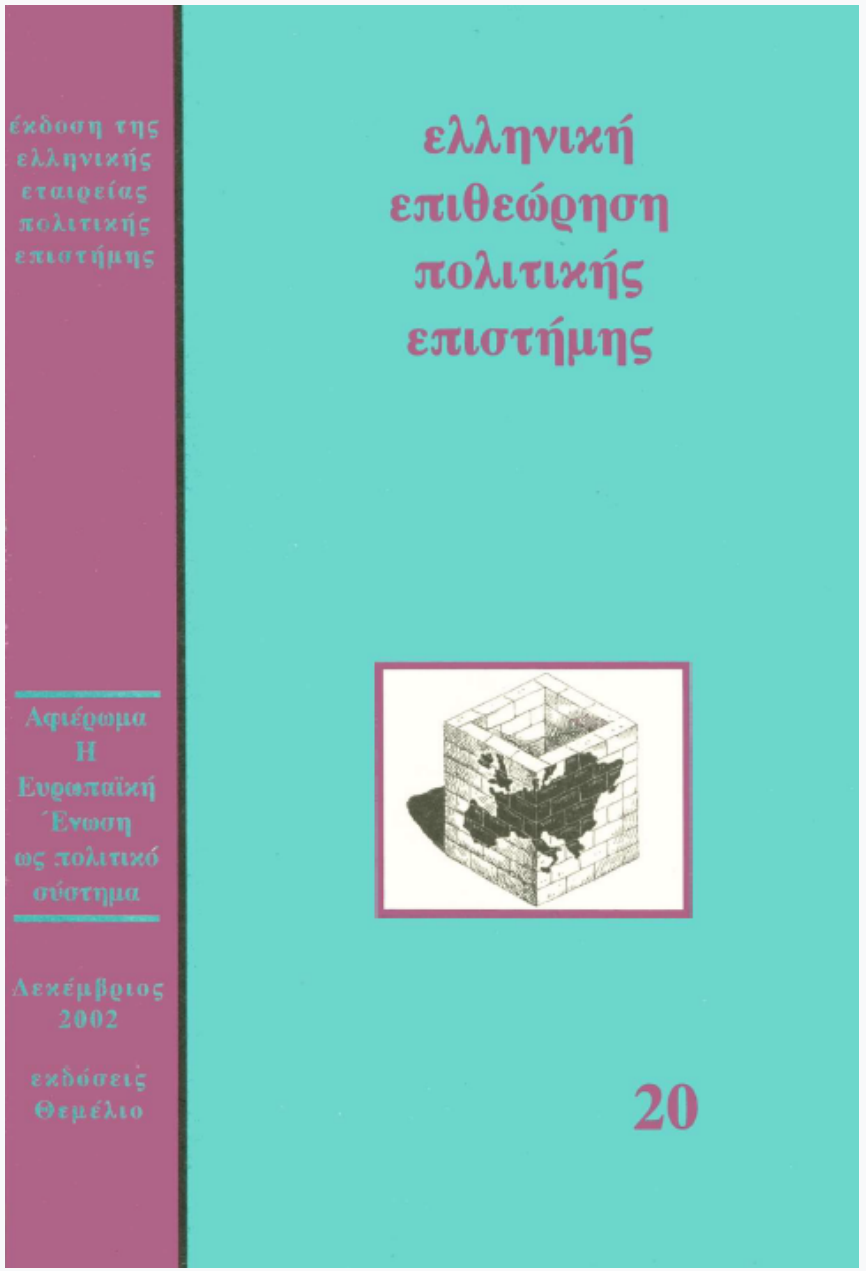

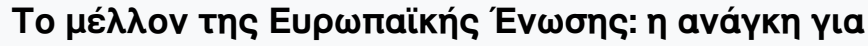

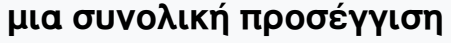

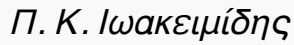

doi: $10.12681 /$ hpsa.14776

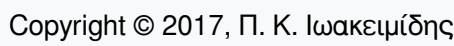

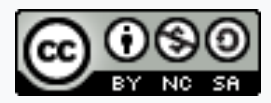

Ađ¿ıı Xpńбnৎ Creative Commons Attribution-NonCommercial-ShareAlike 4.0.

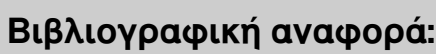

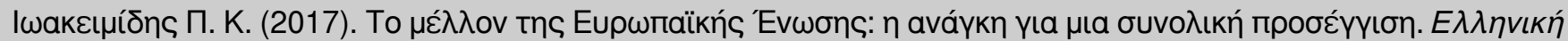

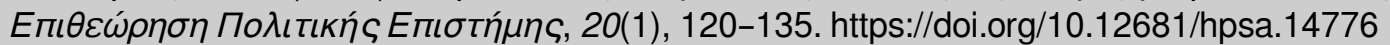




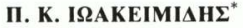

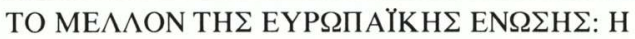 ANAГKH ГIA MIA $\Sigma$ YNOAIKH ПРО $\Sigma$ ЕГГI $\Sigma H$}

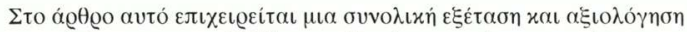

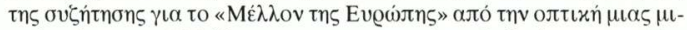

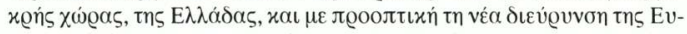

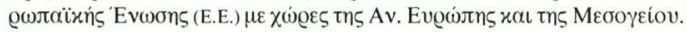

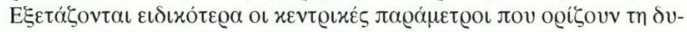

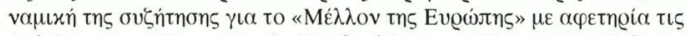

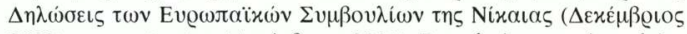

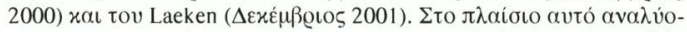

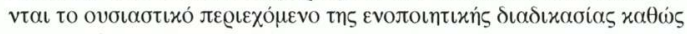

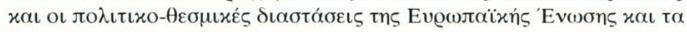

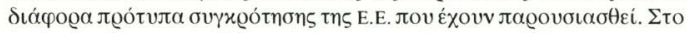

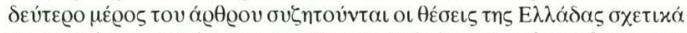

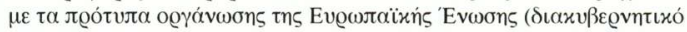

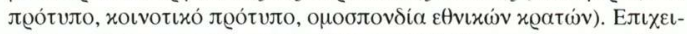

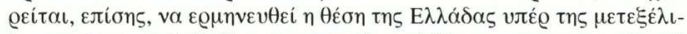

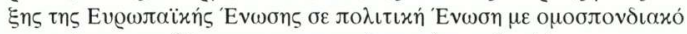

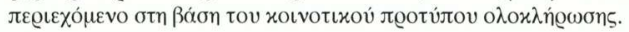

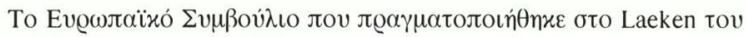

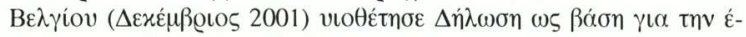

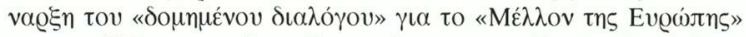

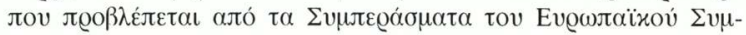

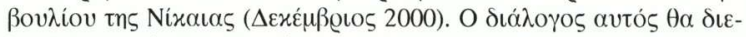

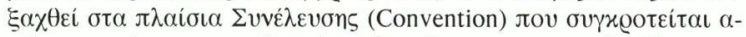

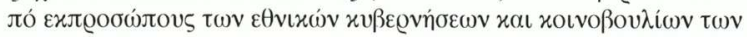

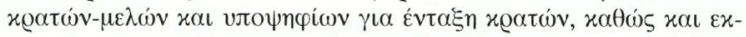

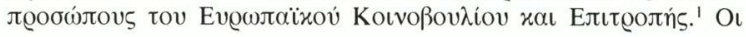

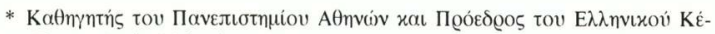

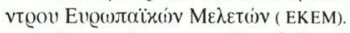

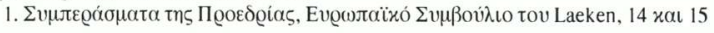




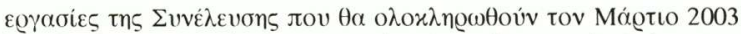

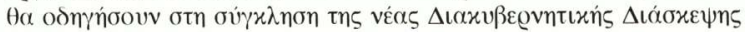

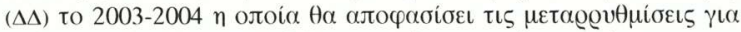

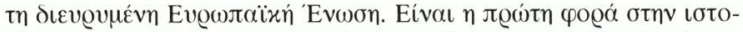

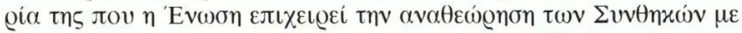
тท

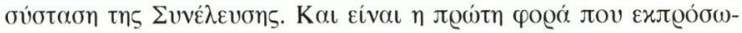

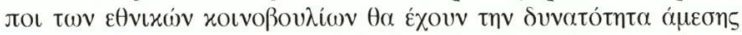

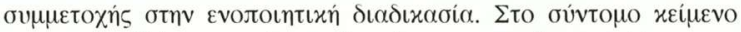

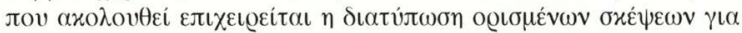

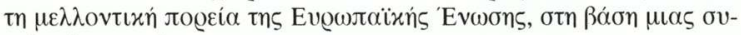

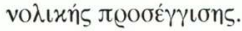

\section{1. $\mathrm{H}$ «META TH NIKAIA» ПPOB $А$ HATIKH}

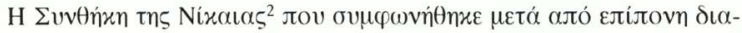

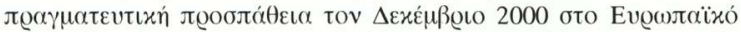

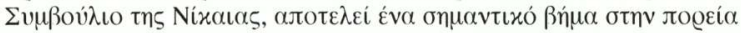

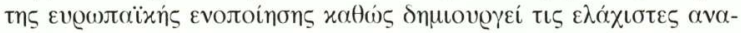

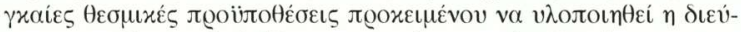

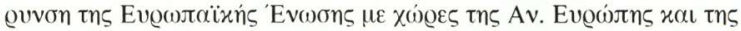

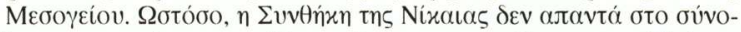

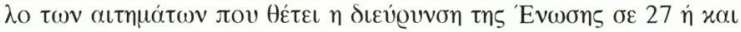

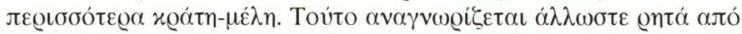

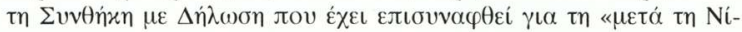

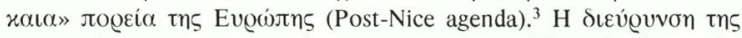

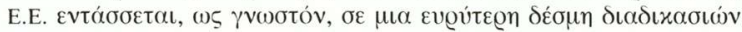

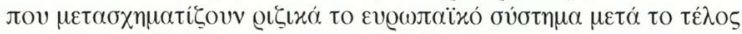

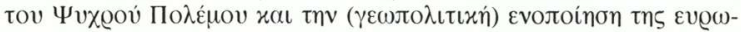

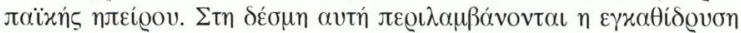

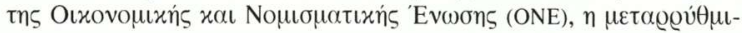

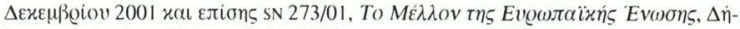
$\lambda \omega \sigma n$ tov Laeken, $15 \Delta \varepsilon x \varepsilon \mu \beta$ oiov 2001.

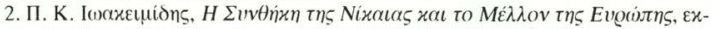

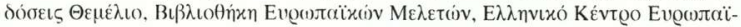

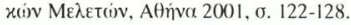

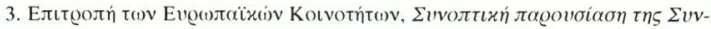

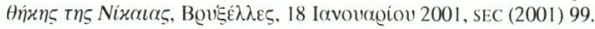




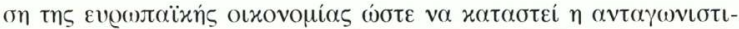

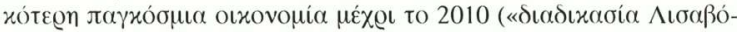

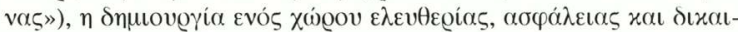

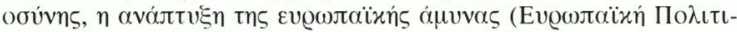

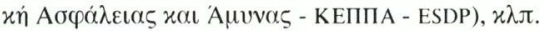

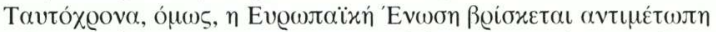

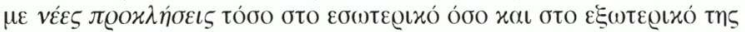

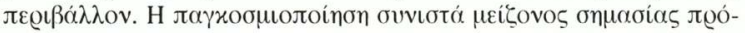

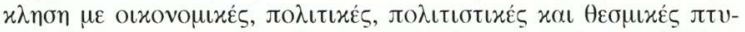

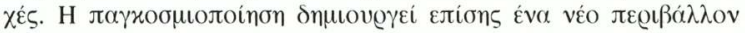

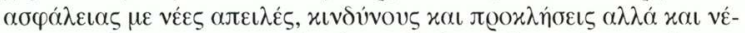

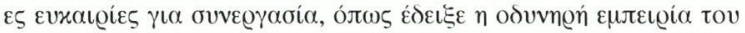

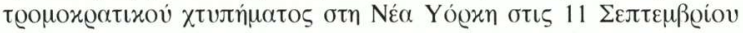

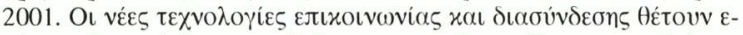

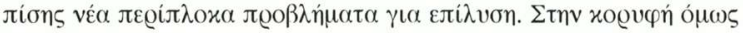

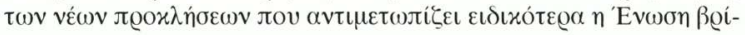

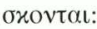

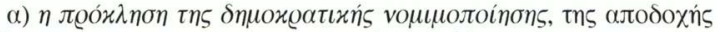

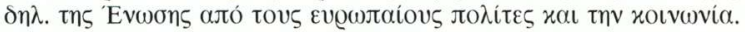

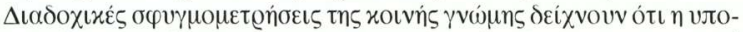

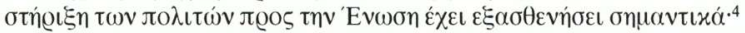

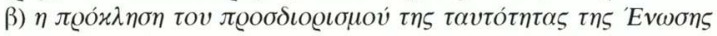

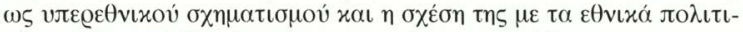

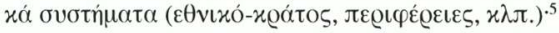

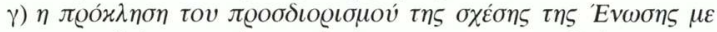

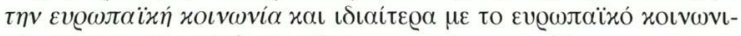

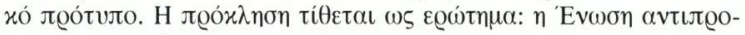

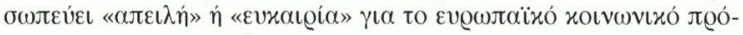

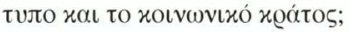

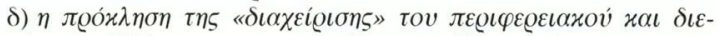

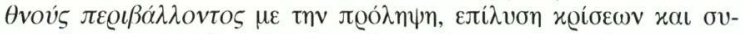

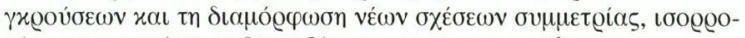

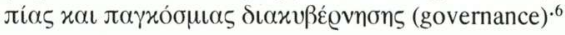

4. European Commission, Eurobarometer Public Opinion in the European Union,

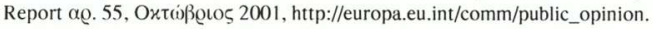

5. П. К. І o.j., б. 128-130.

6. Commission of the European Communities, «European Governance. A White

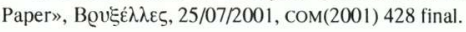




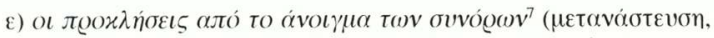

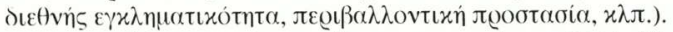

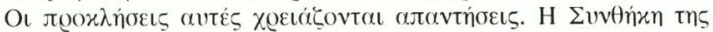

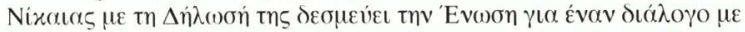

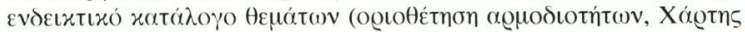

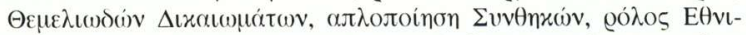

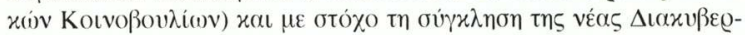

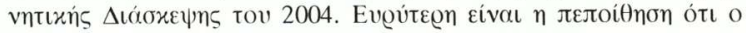

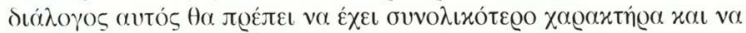

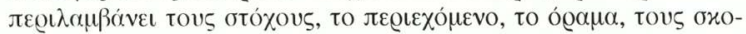

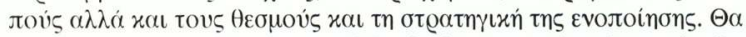

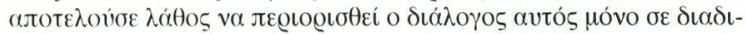

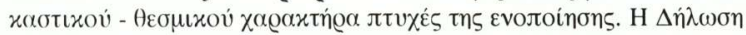

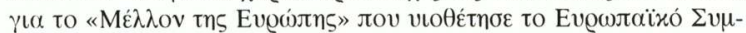

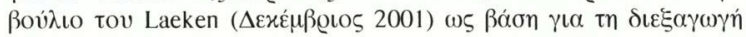

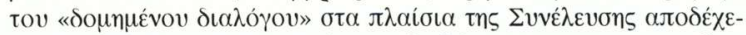

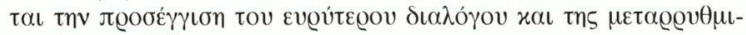
otıxńs agenda. ${ }^{8}$

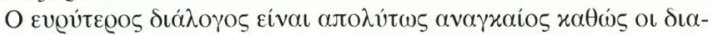

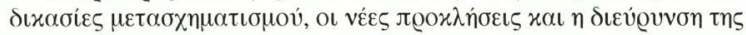

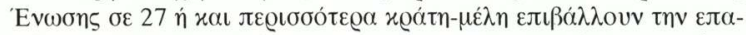

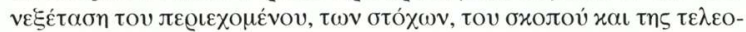

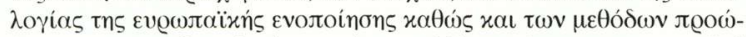

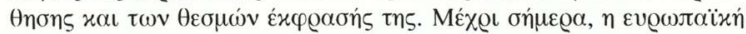

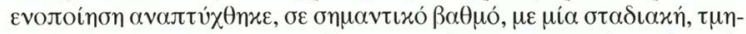

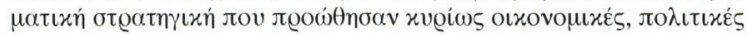

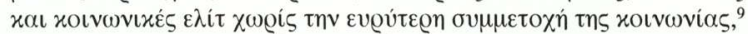

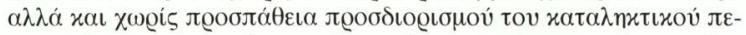

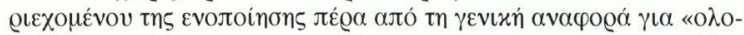

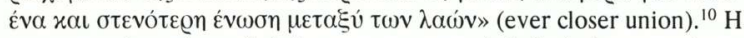

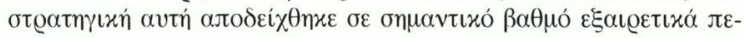

7. EC Official Journal L 344, «Fight Against Terrorism» - Acts adopted by the Council, $28 \Delta \varepsilon x \varepsilon \mu \beta$ ßiov 2001.

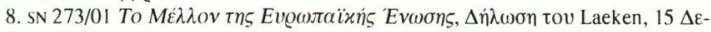

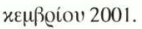

9. Paul Magnette, European Governance and Civic Participation: Can the European Union be politicized?, Jean Monnet Program - Harvard University, w.p. $\alpha \varrho$. $6 / 01$, o. 1-5.

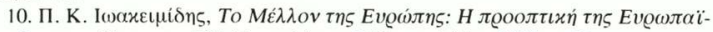

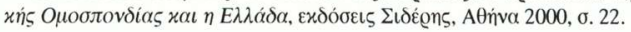




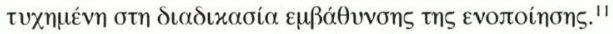

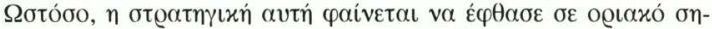

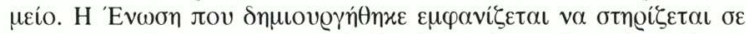

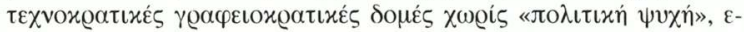

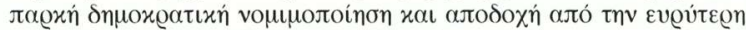

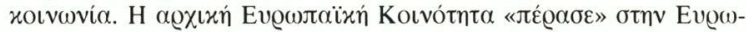

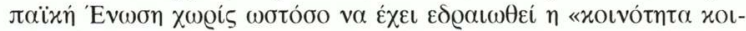

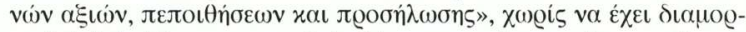

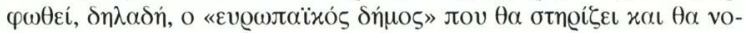

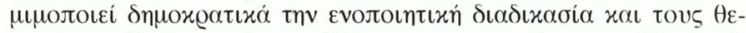

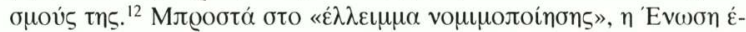

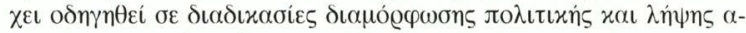

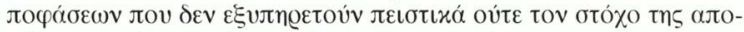

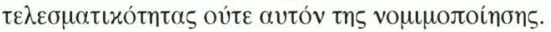

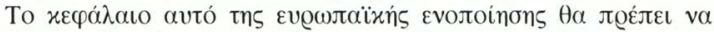

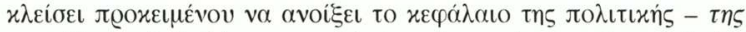

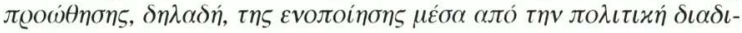

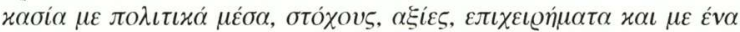

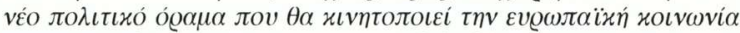

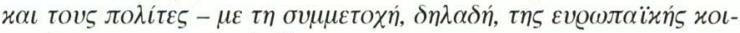
$v \omega v i \alpha \varsigma x \alpha \iota \tau \omega v \pi \circ \lambda \iota \tau \omega v .^{13}$

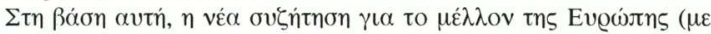

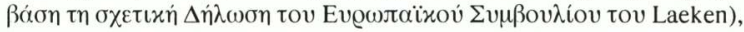

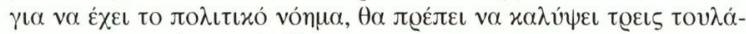

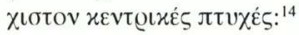

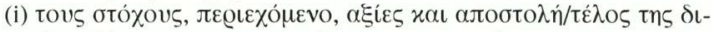

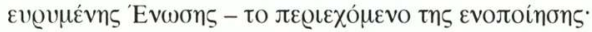

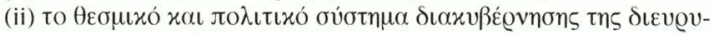

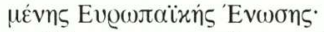

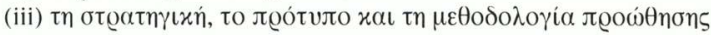

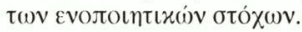

11. David McKay, Designing Europe, Comparative Lessons from the Federal

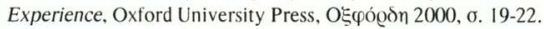

12. Tanja A. Borjel - Thomas Risse. The Post Nice Agenda of the European Union, What's the Problem and how to deal with it, and what to avoid, European University Institute, Robert Schuman Centre, revised draft 03/05/2001, б. 5-10.

13. Peter Ludlow, A View From Brussels A Commentary on the EU 2004 and beyond, 2001, б. 1-2 xa 21-22.

14. П. K. І

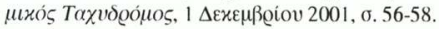




\section{2. ПEPIEXOMENO TH ENOПOIH $\Sigma H \Sigma$}

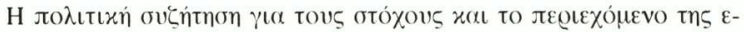

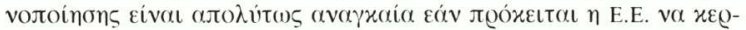

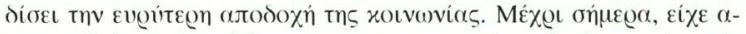

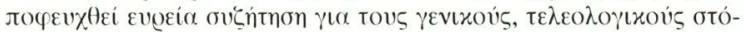

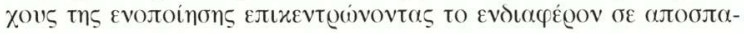

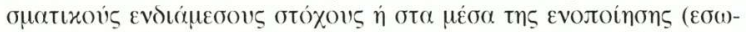

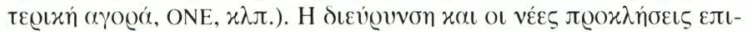

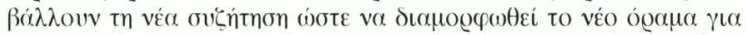

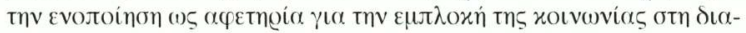

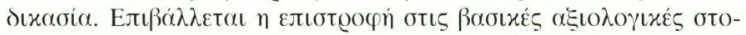

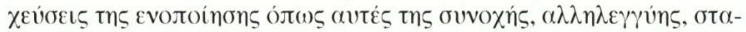

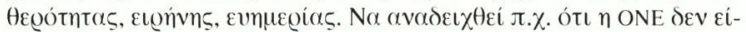

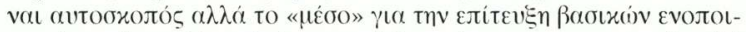

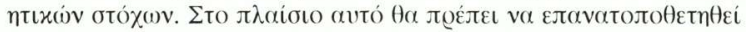

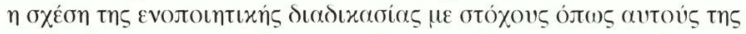

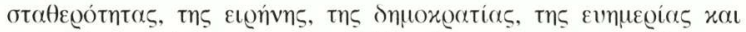

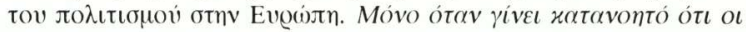

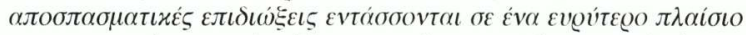

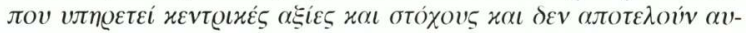

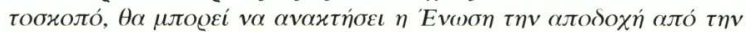

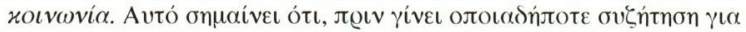

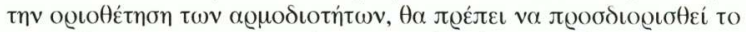

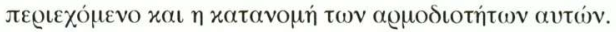

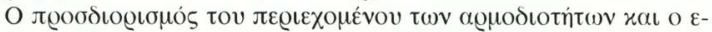

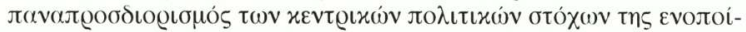

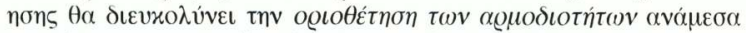

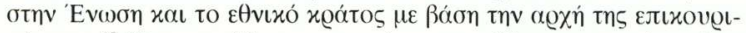

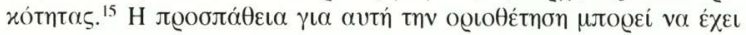

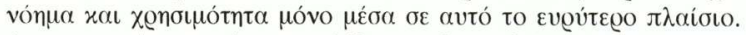

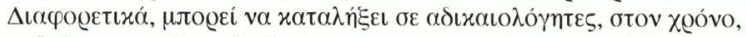

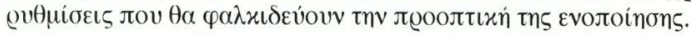

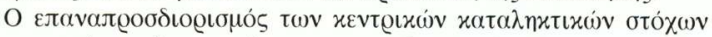

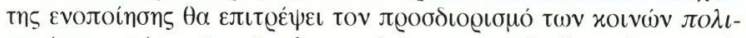

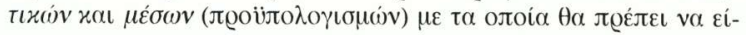

15. David McKay, Designing Europe, Comparative Lessons from the Federal Ex-

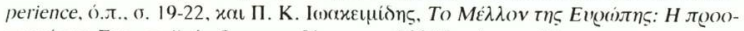

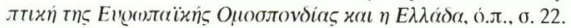




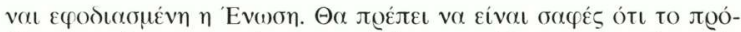

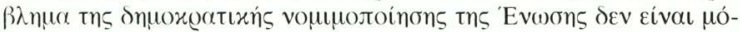

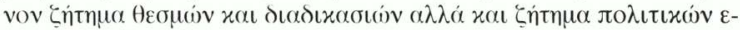

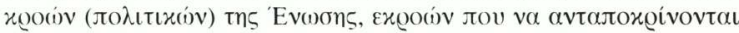

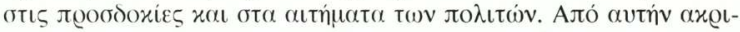

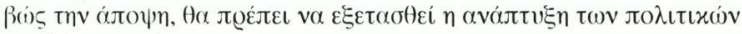

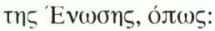

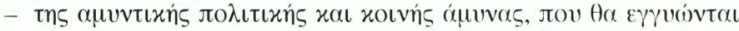

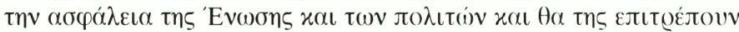

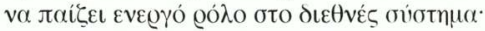

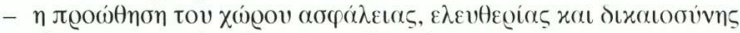

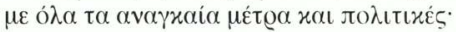

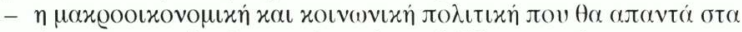

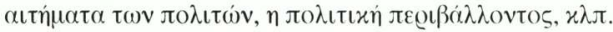

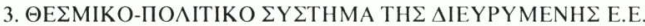

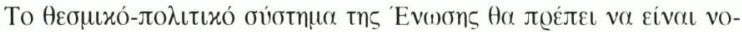

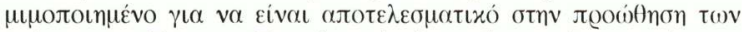

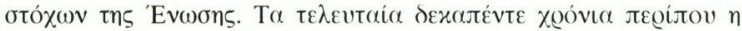

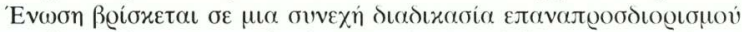

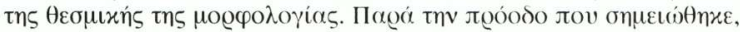

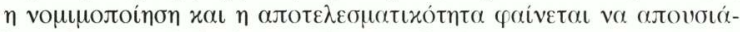

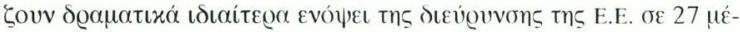

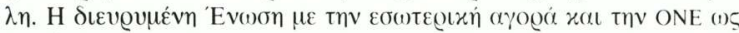

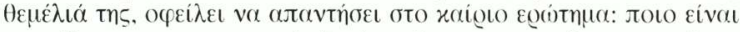

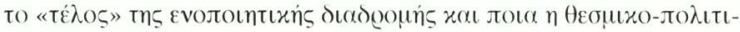

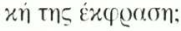

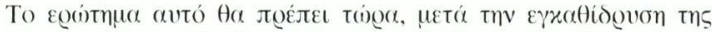

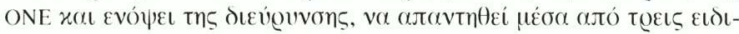

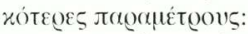

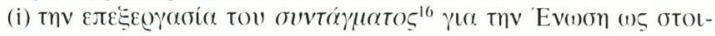

16. Klaus Von Beyme. «Fischer's Move Towards a European Constitution», Jean Monnet Working Paper. (c). 7/00. Symposium: Responses to Joschka Fischer. oto http:// www. jeanmonnetprogram.org/papers/00/00f02011:N-04.html, o. 5. "Governance and Subsidiarity in the European Union: Towards an institutional framework for a European constitution", Permanent Study Group For the European Constitution. In association with The European Institute. South Bank University London 10. 10 February 


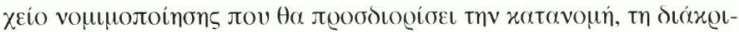

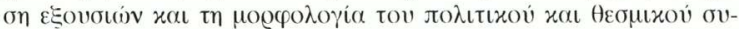

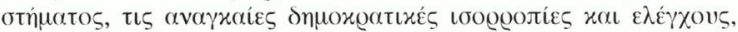

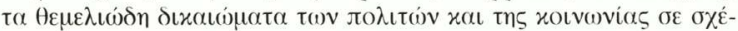

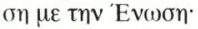

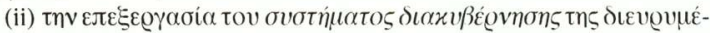

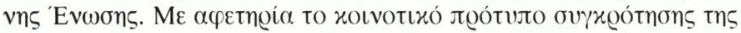

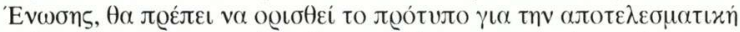

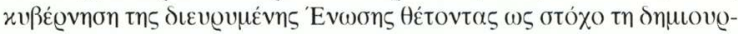

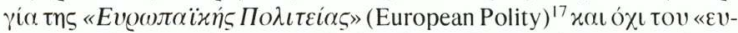

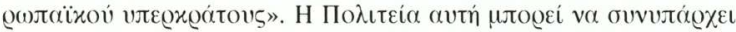

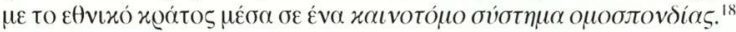

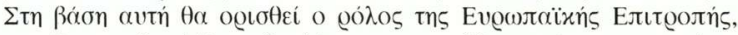

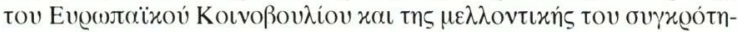

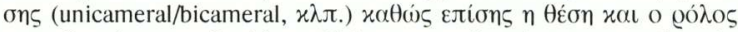

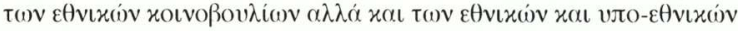

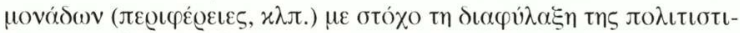

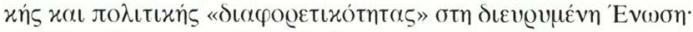

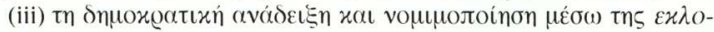

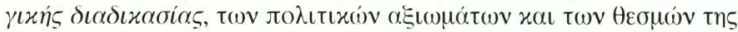

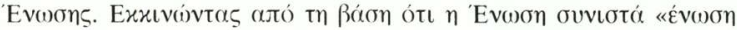

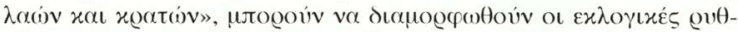

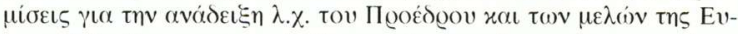

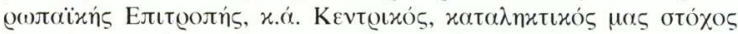

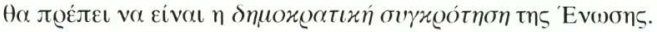

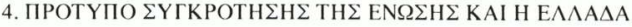

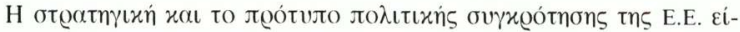

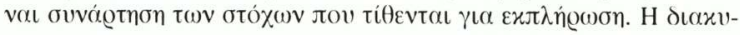

1999, бto http://ig.cs.tu-berlin.de/ev/psg/doc/const93.html, б. 3. R. Koslowski, «Understanding the FU as a Federal Polity", oto T. Christiansen - E.K. Jorgensen - A. Wiener $(\varepsilon \pi \mu$.), The Social Construction of Europe. Sage, Aovoivo 2001, б. 36.

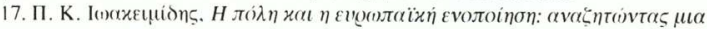

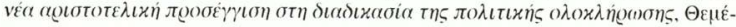

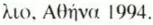

18. Paul Magnette, European Governance and Civic Participation: Can the European Union be politicized?, o.j.. $\sigma .1-5$. 


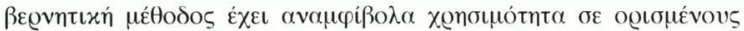

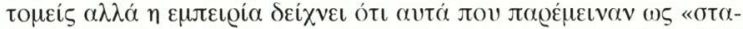

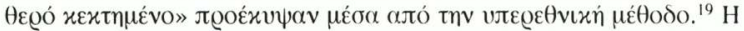

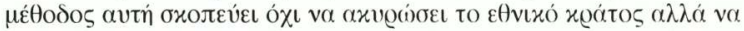

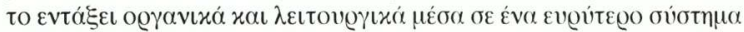

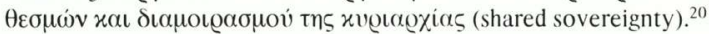

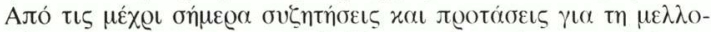

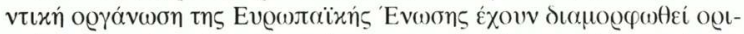

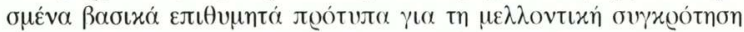

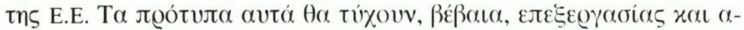

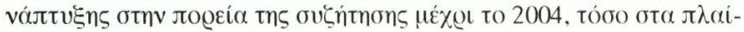

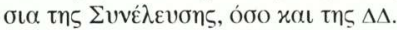

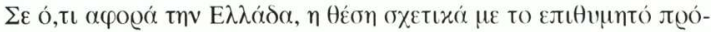

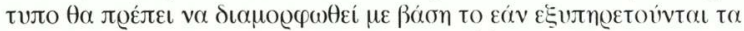

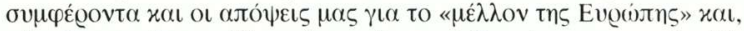

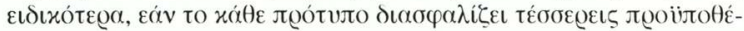

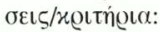

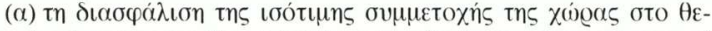

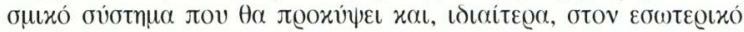

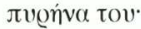

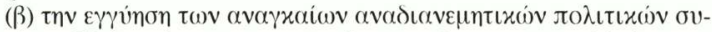

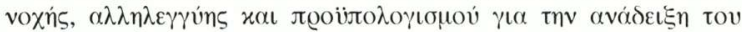

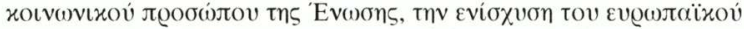

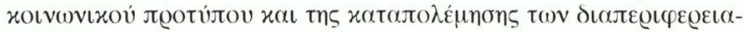

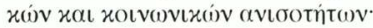

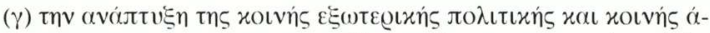

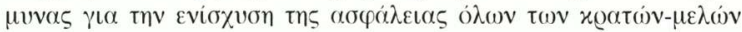

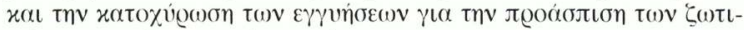

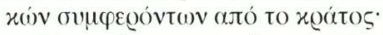

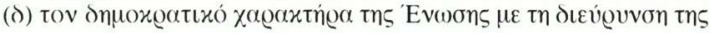

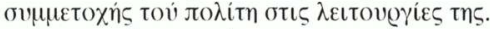

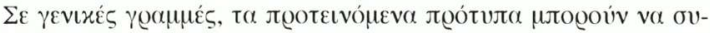

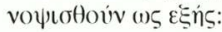

19. Richard E. Baldwin - Erik Berglof - Fransesco Giavaz7i - Mika Widgren, Nice Try: Should the Treaty of Nice be Ratified, Monitoring European Integration II, CEPR, Ioivioc 2001, б. 35-36.

20. Elizabeth Pond, «A New Constitution for the Old Continent», The Washington

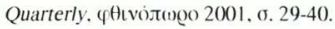




\section{$\alpha . \Delta เ \alpha x$}

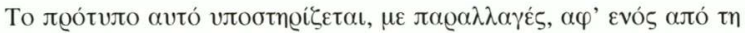

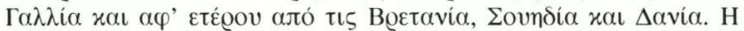

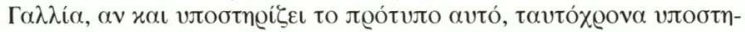

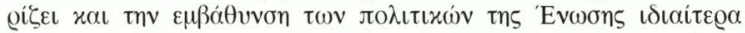

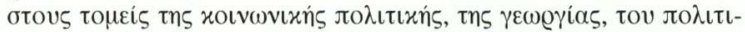

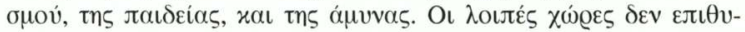

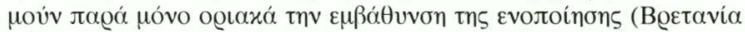
- $\alpha \dot{\mu} \mu v \alpha \alpha)$.

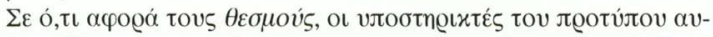

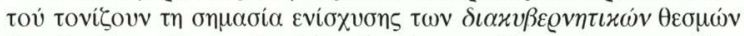

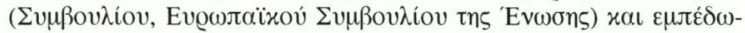

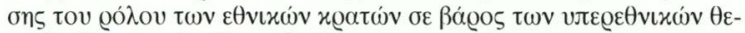

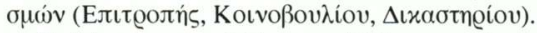

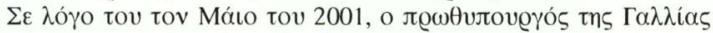

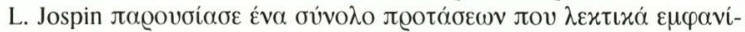

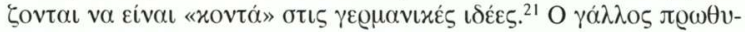

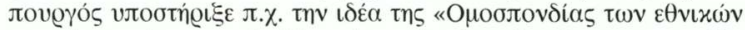

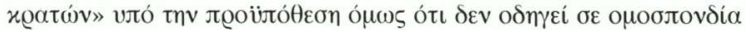

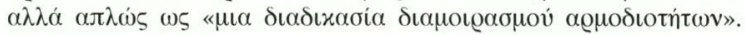

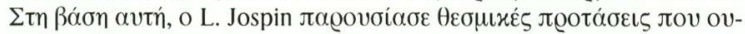

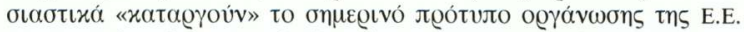

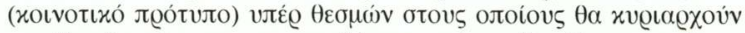

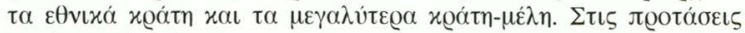

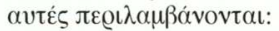

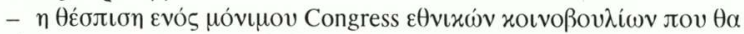

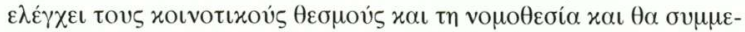

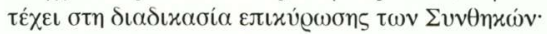

- $\eta \pi \lambda$ п்

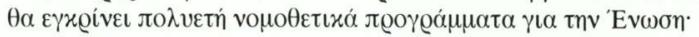

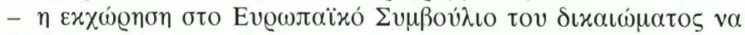

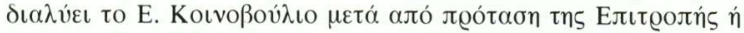

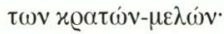

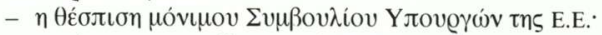

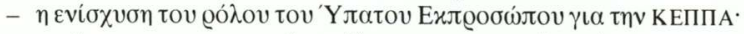

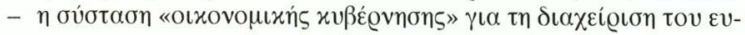

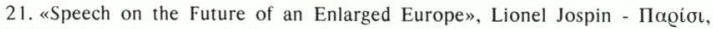
28/05/2001, http://europa.eu.int/futurum/congov_en.htm\#france. 


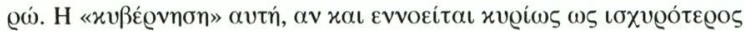

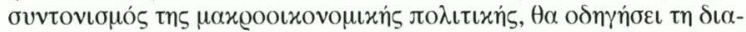

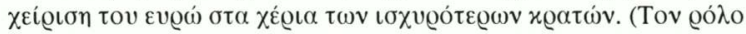

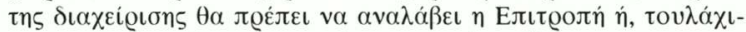

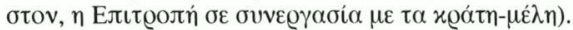

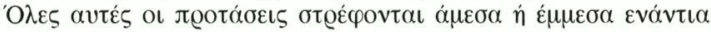

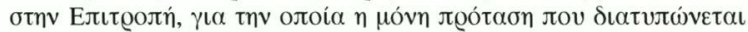

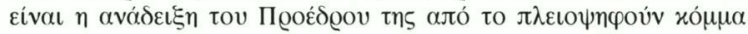

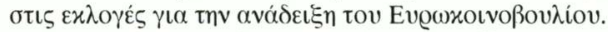

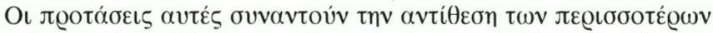

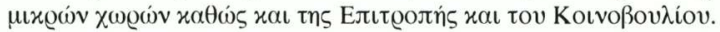

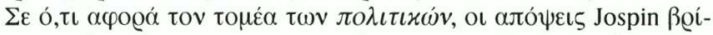

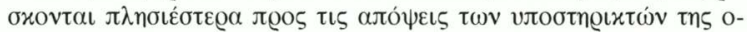

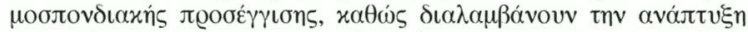

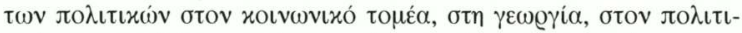

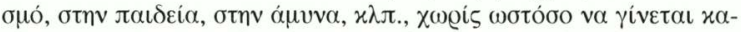

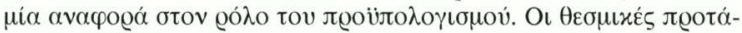

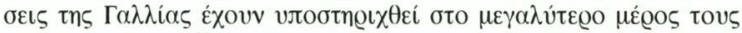

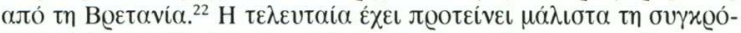

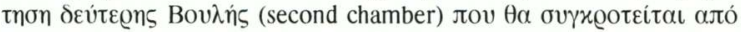

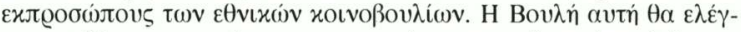

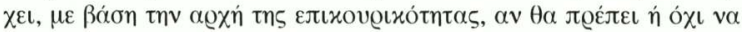

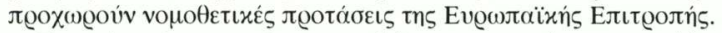

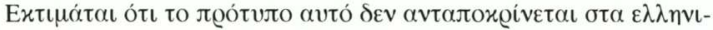

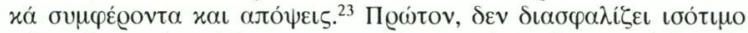

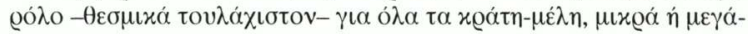

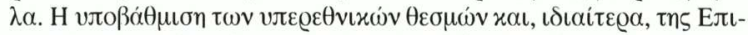

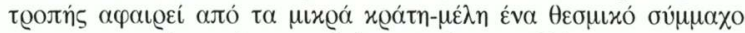

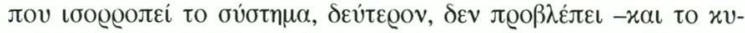

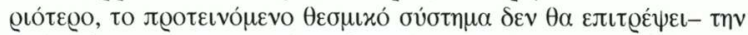

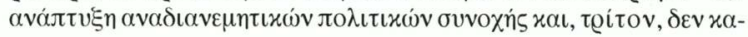

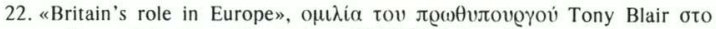
European Research Institute, Birmingham, 23/11/2001, http://europa.eu.int/futurum/ congov_en.htm\#uk.

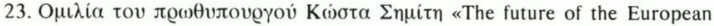

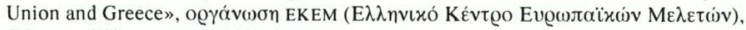

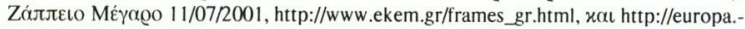

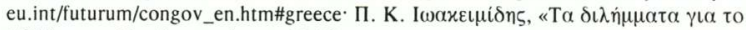

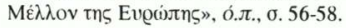




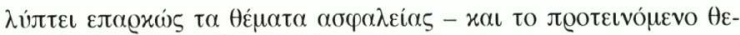

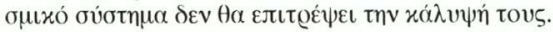

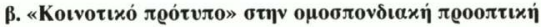

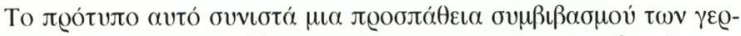

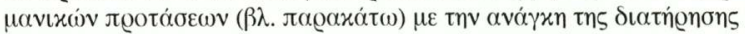

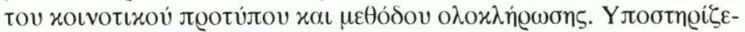

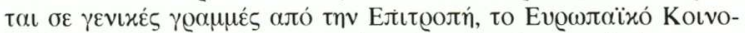

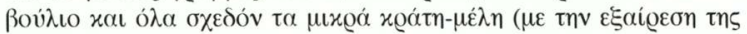
$\Delta \alpha v i \alpha \varsigma x \alpha$ tnऽ $\left.\Sigma o u n d i \alpha \varsigma^{24}\right)$.

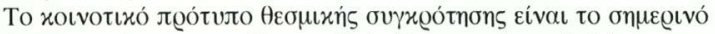

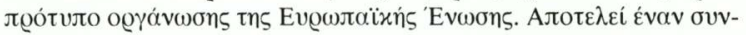

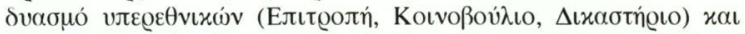

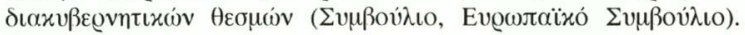

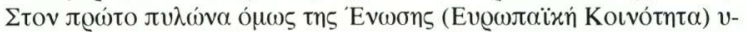

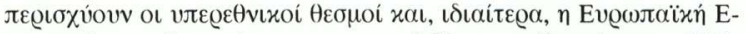

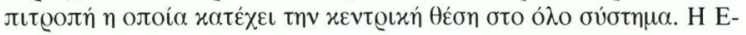

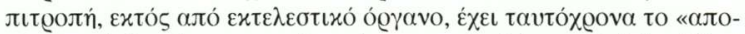

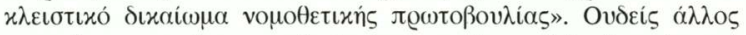

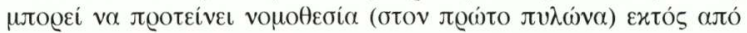

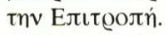

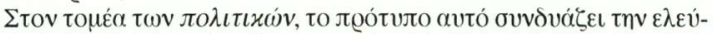

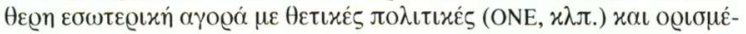

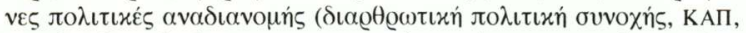

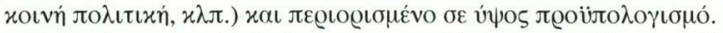

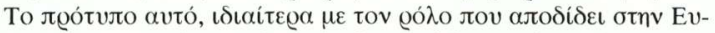

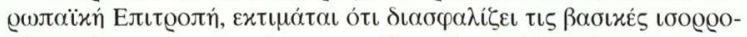

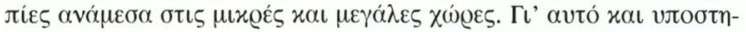

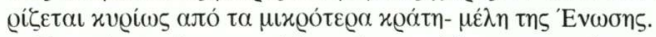

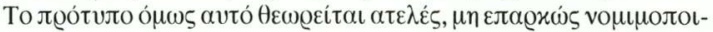

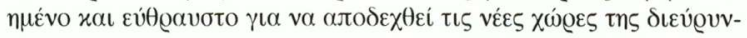

24. The Danish Government's contribution to the debate on the future of Europe,

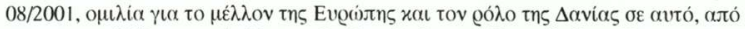

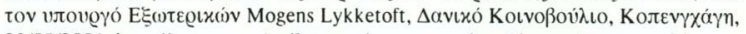
23/08/2001, http://europa.eu.int/futurum/congov_en.htm\#denmark, $x \alpha \iota$ o $\mu$ iti $\alpha$ tov

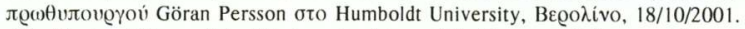

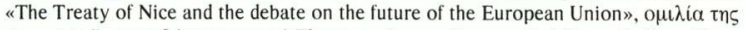

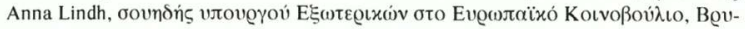

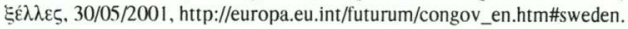




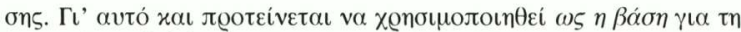

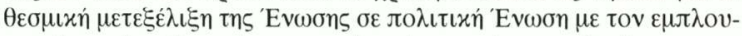

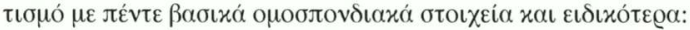

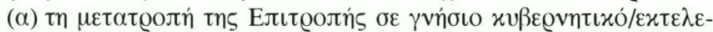

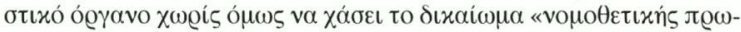

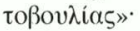

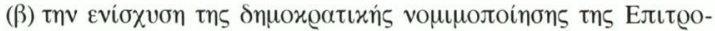

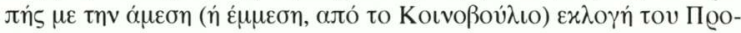

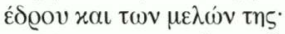

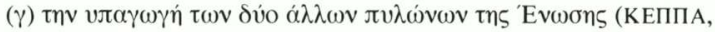

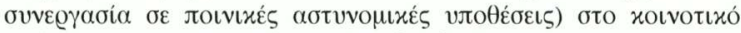

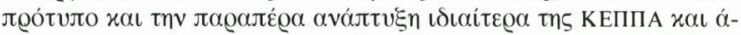

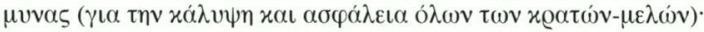

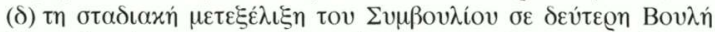

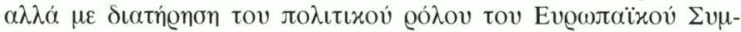
Bovìiov.

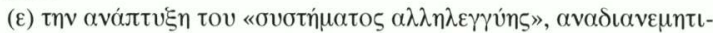

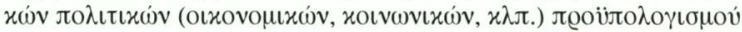

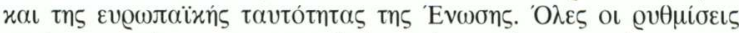

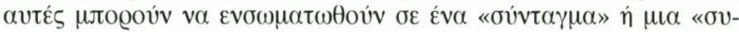

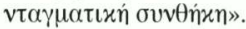

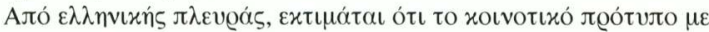

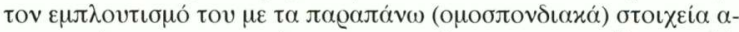

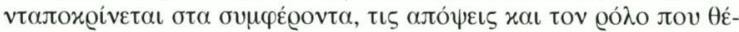

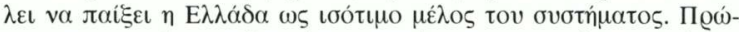

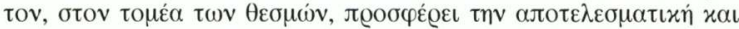

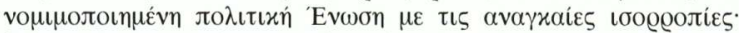

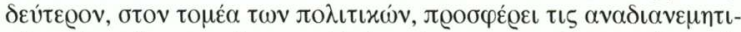

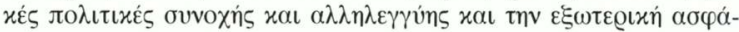

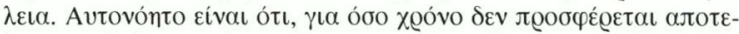

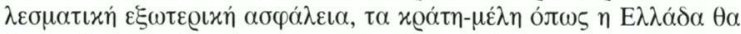

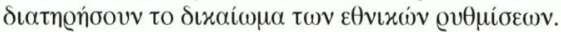

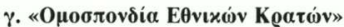

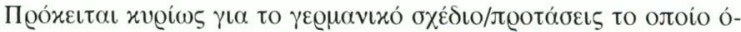

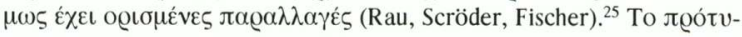

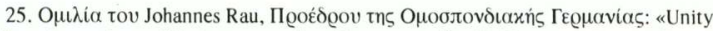
in diversity - what political structure does Europe need?», 7o European Forum, BE@o- 


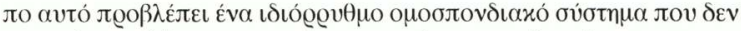

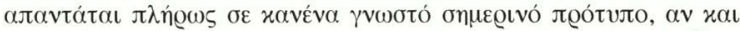

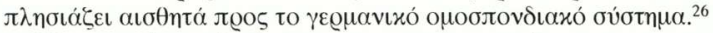

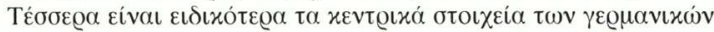
$\pi \varrho \circ \tau \dot{\alpha} \sigma \varepsilon \omega v:^{27}$

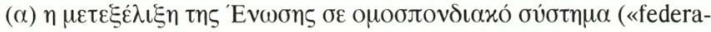

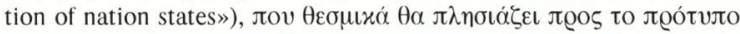

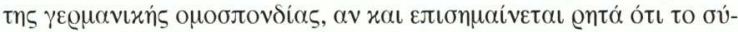

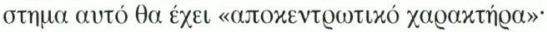

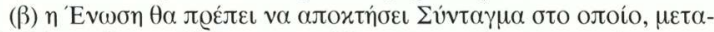

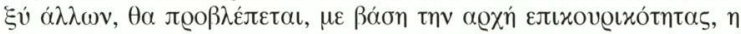

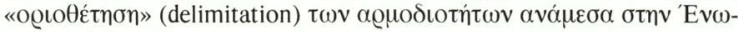

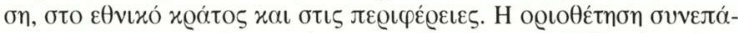

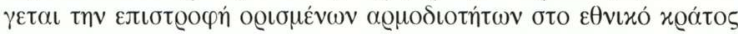

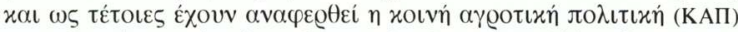

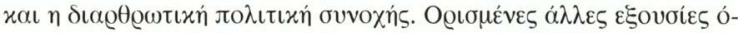

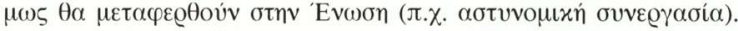

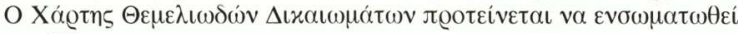

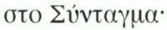

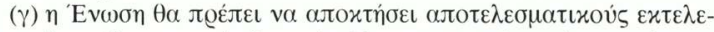

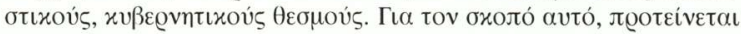

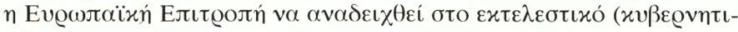
xó) $\sigma \omega ́ \mu \alpha$ tทร 'Evwons.

Aivo, 16/11/2001, xal In the context of the "Tuesdays for Europe» debate on the future of the European Union: minutes of the hearing of Joschka Fischer, $\gamma \varepsilon \varrho \mu \alpha v o v v$ -

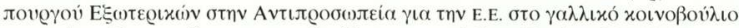

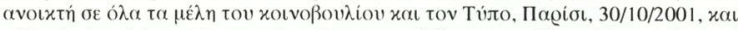

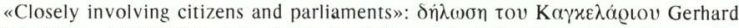
Schröder - 14/06/2001, xal «From Confederacy to Federation - Thoughts on the

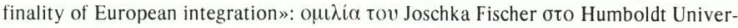
sity, Begonívo, 12/05/2000, raı Plea for a European constitution tov Johannes Rau,

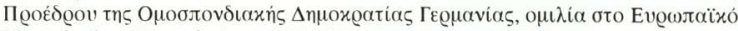

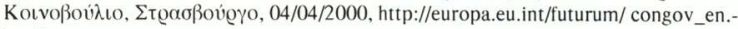
htm\#germany.

26. Dusan Sidjanski, «The federal approach to the european union or the quest for an unprecented european federalism», Gropupement d'Etudes et de Recherches, Notre Europe. Research and Policy Paper $\alpha \varrho$. 14, Ioúl $\operatorname{los} 2001$.

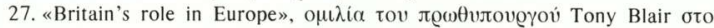
European Research Institute, Birmingham, 23/11/2001, http://europa.eu.int/futurum/ congov_en.htm\#uk. 


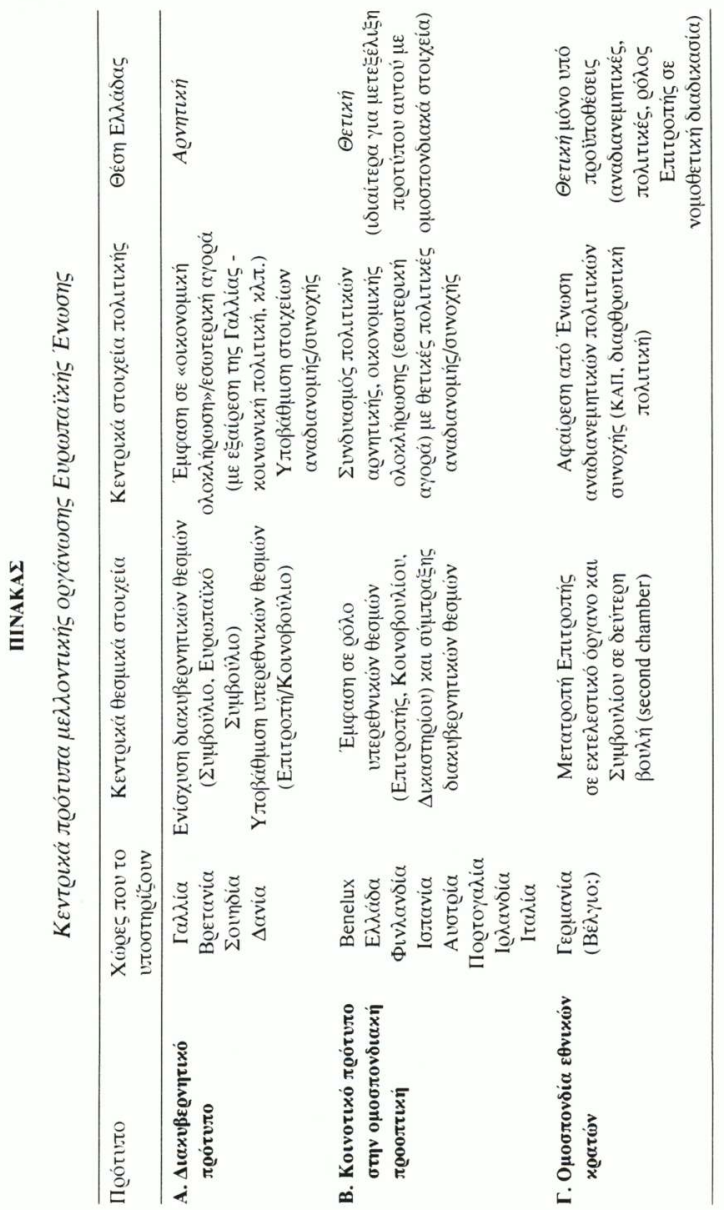




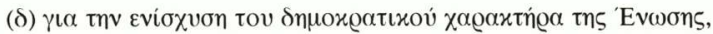

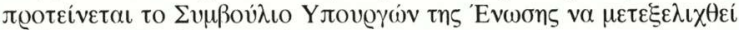

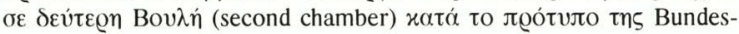

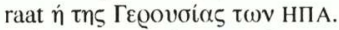

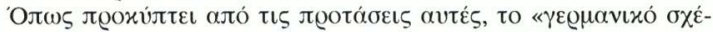

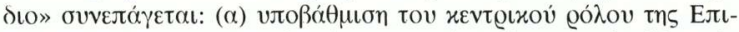

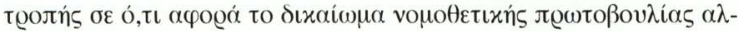

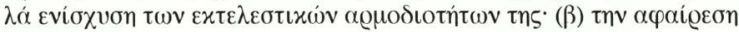

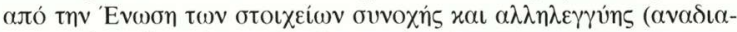

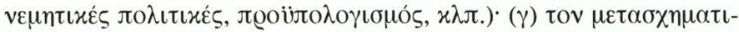

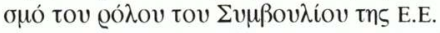

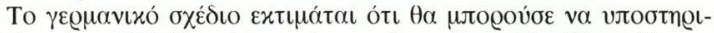

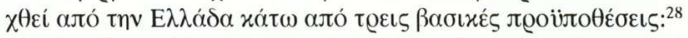

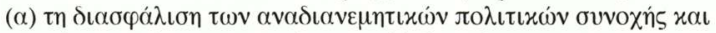

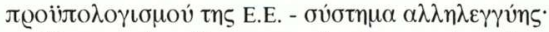

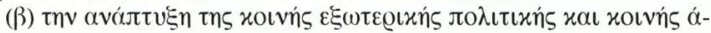

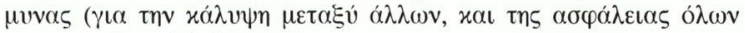

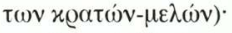

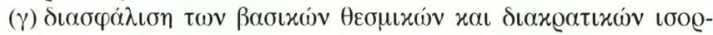

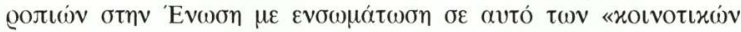

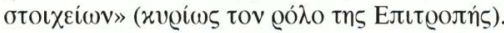

28. Elizabeth Pond, "A New Constitution for the Old Continent», O. $\pi$. 